\title{
Half-Mode Substrate Integrated Waveguide Yagi Array with Low Cross Polarization
}

\author{
Zhao ZHANG ${ }^{1}$, Xiangyu $C A O^{1}$, Jun $G A O^{1}$, Sijia $L I^{1}$, Liming $X U^{2}$ \\ ${ }^{1}$ Information and Navigation College, Air Force Engineering University, Xi'an 710077, China \\ ${ }^{2}$ Science and Technology on Electronic Information Control Laboratory, Chengdu 610036, China
}

bjzhangzhao323@126.com, xiangyucaokdy@163.com,gjgj9694@163.com,1sj051@126.com,10575229@qq.com

Submitted September 23, 2016 / Accepted May 30, 2017

\begin{abstract}
Low cross polarization Yagi array with mirrored arrangement is proposed. First, the half-mode substrate integrated waveguide (HMSIW) and magnetic wall are introduced to realize the miniaturization of Yagi antenna. Simulated results show that the total area of the Yagi antenna is $1.82 \lambda \times 0.57 \lambda$ and the peak gain is about 6.0 7.9 dBi in the $10.5 \%$ relative bandwidth with VSWR less than 2. Then the element arrangement of Yagi array composed by the HMSIW Yagi antenna is analyzed to accomplish cross polarization elimination. It is found that the mirrored arrangement eliminates the far field cross-polar component and leads to the lower cross polarization than the other non-mirrored arrays under the condition that they have almost the same bandwidth, peak gain and beam direction. The low cross polarization four-element array with mirrored arrangement is fabricated and measured, and experimental results agree well with the simulation.
\end{abstract}

\section{Keywords}

Half-mode substrate integrated waveguide, low cross polarization, mirrored arrangement, Yagi array

\section{Introduction}

With the development of satellite communications, antennas achieving compact size, low profile, high gain, specific radiation patterns and ease integration with planar circuits are highly demanded. As a quasi end-fire antenna with a tilted beam in the elevation plane, the microstrip Yagi antenna is one of the candidates for the satellite communications system [1]. The design methods and the performance of microstrip Yagi antennas have been reported since 1990s [2-4]. Based on these antennas, many microstrip Yagi arrays have been developed to extend the performance. By duplicating the microstrip Yagi antenna perpendicular to the projective direction of the tilted beam, linear arrays would be obtained [5-7]. This method of array arrangement is mainly used to achieve a high gain greater than $10 \mathrm{dBi}$. In addition, electronic scanning could be achieved by feeding each element with calibrated phase shifters [6]. Another array arrangement is the crossed Yagi array [8-11], which usually shares a common element at the center. Combined with the switching elements, such as single pole three throw switches and pin diodes, the crossed Yagi arrays have reconfigurable radiation patterns covering the full azimuth plane. These different methods of array arrangement provide more performance features for the Yagi array.

Recently, the half-mode substrate integrated waveguide (HMSIW) and the quarter-mode substrate integrated waveguide (QMSIW) with asymmetrical configurations have been proposed by employing the fictitious quasi magnetic wall in the substrate integrated waveguide (SIW) [12-14]. The HMSIW and QMSIW are only half or quarter size of the SIW but they almost preserve the field distribution of the original SIW. Therefore, the antennas based on the HMSIW and QMSIW not only reduce the antenna size, but also provide more choices for special array arrangements. In [15], an eighth-mode SIW (EMSIW) cavity is formed by bisecting the QMSIW, and then two EMSIW cavities are orthogonally placed to generate the circularly polarized wave [16]. Another compact circularly polarized antenna is proposed by sequential rotation of the isosceles right triangular QMSIW antennas of linear polarization [17]. However, the above two antennas exhibit a different cross polarization, which indicates that the element arrangement of incomplete-mode SIW has a particular effect on the cross polarization.

In this paper, the low cross polarization HMSIW Yagi array with mirrored arrangement is proposed. Firstly, a compact Yagi antenna is designed by introducing the HMSIW and magnetic wall to realize the antenna miniaturization. The total area of the HMSIW Yagi antenna is $1.82 \lambda \times 0.57 \lambda(\lambda$ represents the wavelength at $10 \mathrm{GHz})$. Within the $10.5 \%$ relative bandwidth with VSWR less than 2 , the peak gain is about $6.0 \sim 7.9 \mathrm{dBi}$. Furthermore, three kinds of two-element array and two kinds of four-element arrays with the same distance of array elements but different arrangements are comparatively analyzed to explore the cross polarization elimination. Simulated results show that the symmetrical arrays with mirrored arrangement, in which each element antenna is the mirrored image of the 
neighboring one, have a much lower cross polarization than the other arrays under the condition that they have almost the same bandwidth, peak gain and beam direction. Finally, the four-element array with mirrored arrangement is fabricated and measured, and the experimental results validate the simulation.

\section{Design of HMSIW Yagi Antenna}

The SIW can be regarded as a special rectangular waveguide with a series of slots on the bilateral narrow walls. This feature leads to the result that only $\mathrm{TE}_{n 0}$ modes can exist in SIW [18], [19]. According to the boundary condition on the surface of SIW when the forward wave propagates along the $x$ direction, the surface current density $J_{\mathrm{s}}$ can be expressed as [20]

$$
\boldsymbol{J}_{\mathrm{s}}=\boldsymbol{e}_{\mathrm{n}} \times \boldsymbol{H}=\boldsymbol{e}_{\mathrm{x}} H_{\mathrm{x}}-\boldsymbol{e}_{\mathrm{y}} H_{\mathrm{y}}=\boldsymbol{J}_{\mathrm{x}}+\boldsymbol{J}_{\mathrm{y}} .
$$

As shown in Fig. 1(a), the surface current of SIW for the dominant $\mathrm{TE}_{10}$ mode has two components of $J_{x}$ and $J_{y}$. They are symmetrically distributed along the A-A' plane and the component $J_{y}$ has the opposite flowing direction at two sides of A-A'. It also can be found that the current density has its maximum at the A-A' plane. So the fictitious magnetic wall is available for the A-A' plane. After cutting the A-A' plane, the SIW becomes HMSIW which keeps half of the surface current distribution of the dominant mode as shown in Fig. 1(b). The current density has its maximum at the magnetic wall B-B'. It should be pointed out that the resonant frequencies of the HMSIW are slightly shifted up compared with the corresponding SIW one due to the fringing fields of the non-ideal magnetic wall B-B'.

In this paper, we introduce the HMSIW as the driven element of Yagi antenna. The configuration of the proposed HMSIW Yagi antenna is shown in Fig. 2(a). It consists of a driven element $\mathrm{D}$, a coupling patch $\mathrm{C}$ and two director elements (D1 and D2). All the metal patches are printed on the upside of Rogers RT/duroid 5880 substrate

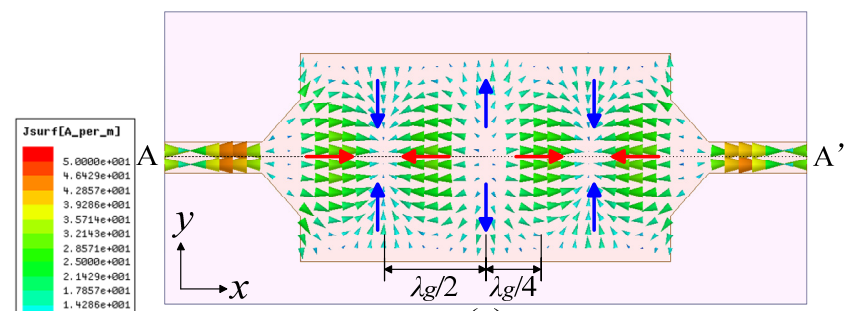

(a)

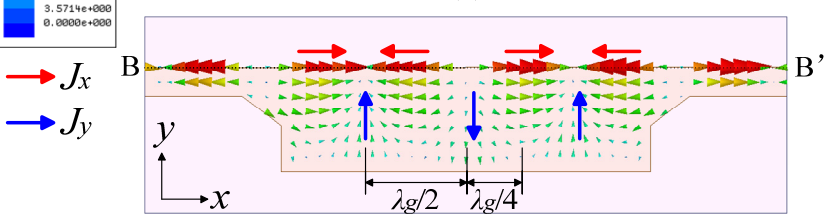

(b)

Fig. 1. Comparison of surface current distribution in (a) SIW and (b) HMSIW.

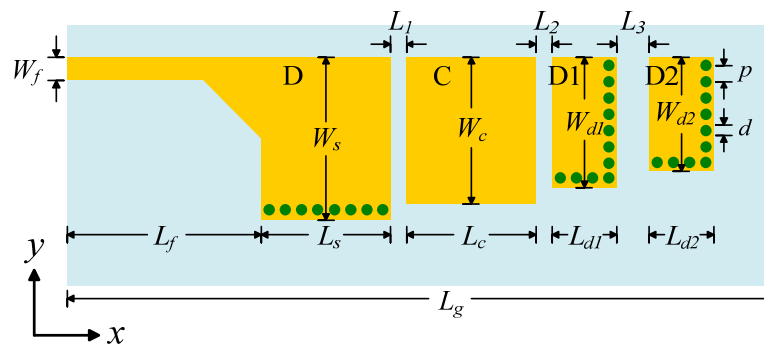

(a)

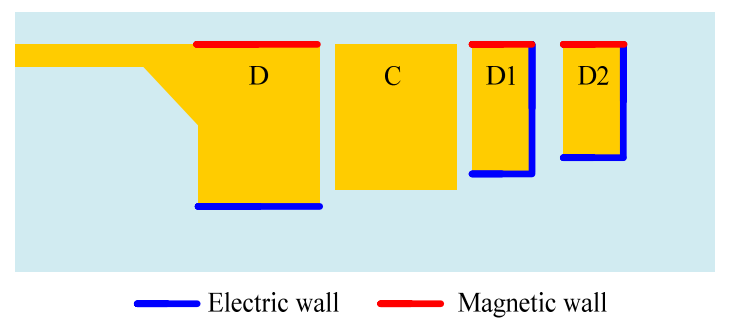

(b)

Fig. 2. Configuration of the HMSIW Yagi antenna. (a) Top view. (b) Equivalent model with electric and magnetic wall

\begin{tabular}{|c|c|c|c|}
\hline Parameters & Value & Parameters & Value \\
\hline$W_{\mathrm{s}}$ & 11.3 & $L_{\mathrm{s}}$ & 7.4 \\
\hline$W_{\mathrm{c}}$ & 10.0 & $L_{\mathrm{c}}$ & 8.0 \\
\hline$W_{\mathrm{d} 1}$ & 8.8 & $L_{\mathrm{d} 1}$ & 4.3 \\
\hline$W_{\mathrm{d} 2}$ & 8.3 & $L_{\mathrm{d} 2}$ & 4.3 \\
\hline$W_{\mathrm{g}}$ & 17 & $L_{\mathrm{g}}$ & 54.6 \\
\hline$L_{1}$ & 0.15 & $p$ & 1 \\
\hline$L_{2}$ & 0.55 & $d$ & 0.6 \\
\hline
\end{tabular}

Tab. 1. Parameters of the proposed antenna (Unit: millimeter).

( $\varepsilon_{\mathrm{r}}=2.2$ and $\left.\tan \delta=0.001\right)$ with thickness $h$ and the underside is a copper ground plane. The driven element $\mathrm{D}$ is a part of HMSIW which works under the dominant $\mathrm{TE}_{10}$ mode. Its width $W_{\mathrm{s}}$ determines the cutoff frequency of HMSIW as well as the working band of the proposed antenna. Due to the period distribution of the standing wave, the length of HMSIW can be flexible. Here, the length $L_{\mathrm{s}}$ of driven element is optimized between $\lambda_{\mathrm{g}} / 4$ and $\lambda_{\mathrm{g}} / 2$. The operating principles of the coupling patch and director elements are similar to those of the magnetic Yagi antenna in [4]. The coupling patch is utilized to improve the impedance matching when its resonant frequency is close to that of HMSIW [21]. Also, it functions as a bridge that guides the electromagnetic energy to the director element so as to radiate forward. The director elements with two edges shorted by plated through holes (PTHs) work as magnetic dipoles to increase the gain and the front-to-back ratio. All the PTHs in HMSIW and the director elements should meet the equations $0.05<p / \lambda_{\mathrm{c}}<0.25$ where $p$ is the distance between PTHs and $\lambda_{\mathrm{c}}$ is the cutoff wavelength [22], so that they can be equivalent to electric wall and leakage losses are negligible. Parametric analyses were conducted using Ansoft HFSS and the optimized dimensions are listed in Tab. 1 .

What makes the proposed antenna asymmetrical and compact is the introduction of magnetic wall. Due to the 
symmetrical electric field distribution at both sides of the driven-director axis in [4], the magnetic wall is introduced. Figure 2(b) shows the equivalent model with electric and magnetic walls. The PTHs are equivalent to the electric walls. Meanwhile, the open sides (shown in red) of the driven element and director elements are approximately regarded as magnetic walls. By means of image theory, this arrangement has a pronounced effect on the antenna miniaturization with radiation characteristics almost preserved. The width of driven element is reduced from $1.05 \lambda$ (in [4]) to $0.38 \lambda$, and the widths of director elements are reduced to $0.29 \lambda$ and $0.28 \lambda$ respectively. Thus, the total area of the antenna is reduced to $1.82 \lambda \times 0.57 \lambda$.

Figure 3 shows the inside electric field distribution at $10 \mathrm{GHz}$. It can be seen that the PTHs work as electric wall and the electric field distribution is restricted. For the driven element D and director elements (D1 and D2), the electric field reaches the maximum along the open sides, which indicates that the open sides are approximately equivalent to magnetic wall. It should be noted that the magnetic wall is strictly a quasi-one. Outside the magnetic wall, there exists fringing field which will generate influence on cross polarization. It is also found that the coupling between element D2 and D1 is relatively weak. Here, element D2 has an important influence on beam steering angle and $\mathrm{F} / \mathrm{B}$ ratio. And properly increasing director elements will generate lager beam steering angle and $\mathrm{F} / \mathrm{B}$ ratio.

Figure 4 shows the simulated 3-D radiation pattern at $10 \mathrm{GHz}$. Obviously, the antenna has a tilted beam and the main beam points toward the low elevation direction.

The simulated results of reflection coefficient $S_{11}$ and peak gain are shown in Fig. 5. The working band of $S_{11}<-10 \mathrm{~dB}$ is $9.5 \sim 10.6 \mathrm{GHz}$ (the relative bandwidth is $10.5 \%$ with VSWR less than 2). The peak gain is about 6.0 7.9 $\mathrm{dBi}$ in the working band.

Figure 6 presents the simulated radiation patterns at 9.5, 10 and $10.6 \mathrm{GHz}$ in elevation plane and azimuth plane. The elevation plane and the azimuth plane represent the xoz plane and the xoy plane respectively according to the Cartesian coordinate in Fig. 4. In the elevation plane, the co-polarization is vertical polarization and the peak gain direction occurs around $40^{\circ}$, which presents a tilted beam similar to that of conventional microstrip Yagi antenna. Within the direction of $0^{\circ} \sim 90^{\circ}$, the cross polarization is lower than $-15 \mathrm{~dB}$ and the cross polarization isolation (the difference between co- and cross-polarization) is greater than $14 \mathrm{~dB}$. In the azimuth plane, the main beam occurs at the end-fire direction and the cross polarization isolation is greater than $10 \mathrm{~dB}$. Meanwhile, both of the co-polarization and cross-polarization radiation patterns are asymmetrical along the $0^{\circ}-180^{\circ}$ in azimuth plane due to the asymmetrical antenna structure. In addition, the radiation efficiency is simulated and results show that the efficiency is 0.964 , 0.989 and 0.937 at $9.5,10$ and $10.6 \mathrm{GHz}$, respectively. So the loss is very small and the antenna has a relatively high efficiency.

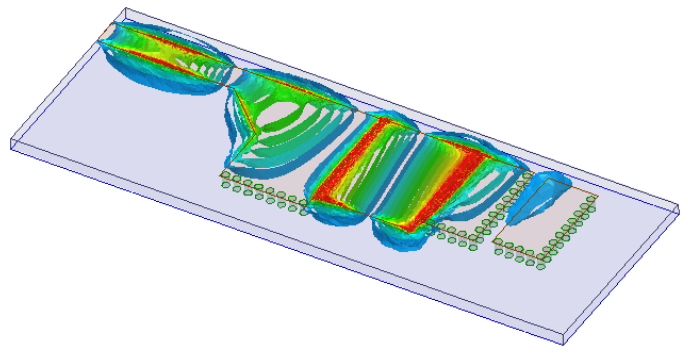

Fig. 3. Electric field distribution at $10 \mathrm{GHz}$.

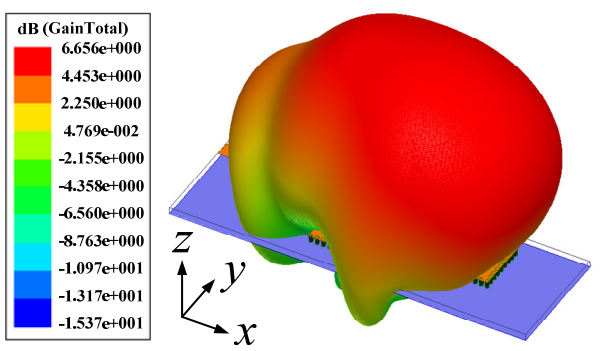

Fig. 4. Simulated 3-D radiation pattern at $10 \mathrm{GHz}$.

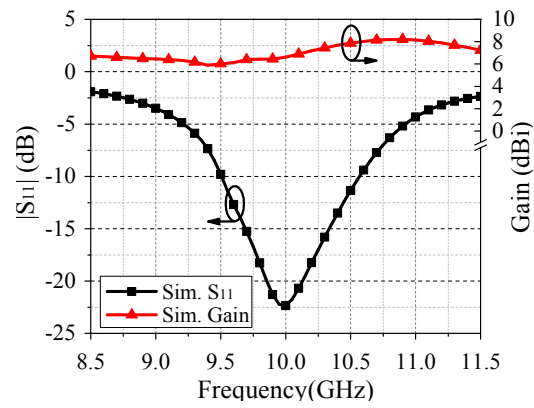

Fig. 5. Simulated $S_{11}$ and peak gain of the proposed antenna.

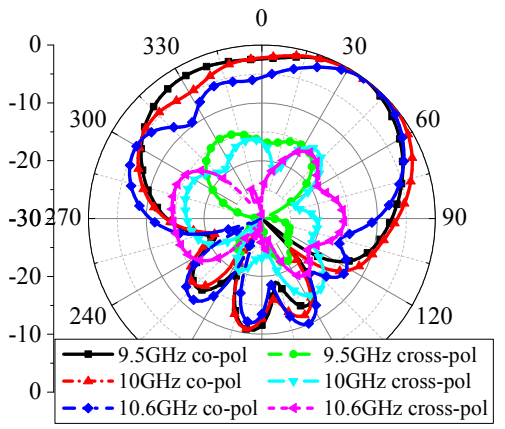

(a)

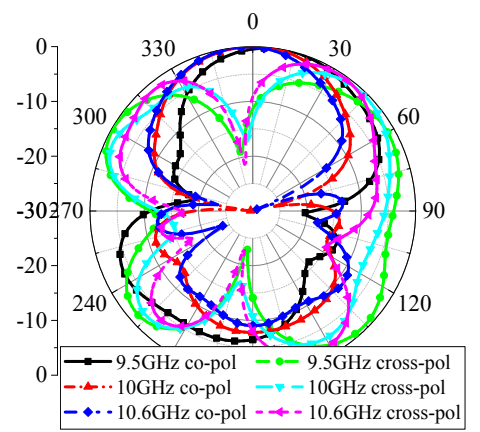

(b)

Fig. 6. Simulated radiation patterns.

(a) Elevation plane. (b) Azimuth plane. 


\section{Analysis of Two-element Yagi Arrays}

Due to the asymmetrical structure and field distribution, the Yagi arrays composed by the HMSIW Yagi antenna with different arrangements have different radiation characteristics. We analyzed the characteristics of three kinds of two-element Yagi array. As shown in Fig. 7, the relative positions of array element are different. The array (I) is an asymmetrical structure and array elements have the same direction of the open sides. The arrays (II) and (III) are mirrored symmetrical structures, and adjacent elements have the opposite direction of the open sides. Differently, the open side direction in array (II) is contrary to the other, while the one in array (III) is facing to the other.

In view of the fringing wave around the open sides, the distances between array elements should be optimized respectively. Namely, array (II) with the contrary direction of open sides should have the minimum distance between array elements, while array (III) with the facing direction of open sides should have the maximum one and array (I) should have the middle one, so as to improve the coupling effect. Here, in order to comparatively analyze the radiation characteristics, we have selected the same distances between arrays elements for the three arrays, and they are $\left.d x_{i}\right|_{i=1,2,3}=17 \mathrm{~mm}$ (about $0.57 \lambda$ ).

The simulated results of reflection coefficient and peak gain are shown in Fig. 8. It can be found that the three arrays have the same curve trend of peak gain, and the peak gain increases by $2.5 \sim 3.0 \mathrm{dBi}$ compared with the single Yagi antenna. As to the reflection coefficient, the array (I) and (II) has the same curve trend and bandwidth, while the array (III) has a slightly wider bandwidth.

Figure 9 shows the radiation patterns at $10 \mathrm{GHz}$. In elevation plane, the co-polarization patterns still coincide with each other and the beam directions point at $40^{\circ}$ at the same time. In azimuth plane, the array (III) has the maximum beam width, while the array (II) has the minimum one. On the other hand, they all have similar cross polarization radiation patterns. It should be pointed out that the difference between beam widths can be eliminated by adjusting the distance between array elements. In a word, although the three arrays have completely different arrangements, they exhibit a remarkable similarity in peak gain, reflection coefficient, co- and cross-polarization radiation pattern in azimuth plane, and co-polarization radiation pattern in elevation plane.

However, what makes one array distinctly different from the others is the cross polarization in elevation plane. For the array (II) and (III), the cross polarization is below $-50 \mathrm{~dB}$ within the direction of $0^{\circ} \sim 90^{\circ}$ (the cross polarization isolation is greater than $45 \mathrm{~dB}$ ), which is much lower than that of the array (I). The difference of cross polarization can be attributed to the particular element arrangement. With the mirrored arrangement, each element in array (II) and (III) has the mirrored current distribution of its neigh-

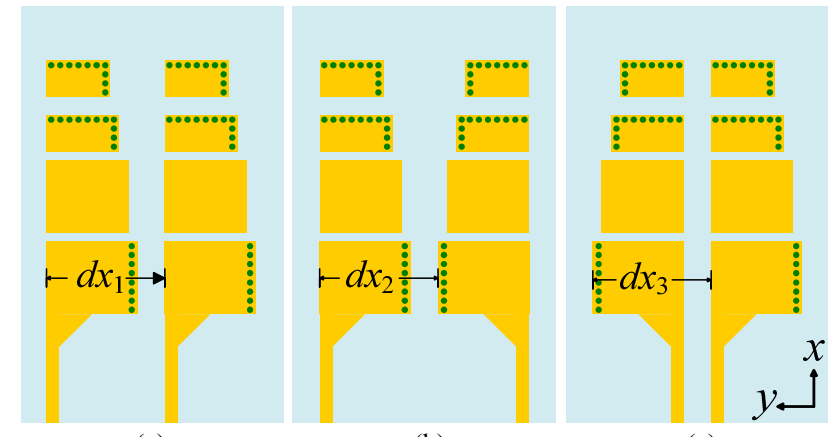

(a)

(b)

(c)

Fig. 7. Two-element arrays. (a) Array (I). (b) Array (II). (c) Array (III).

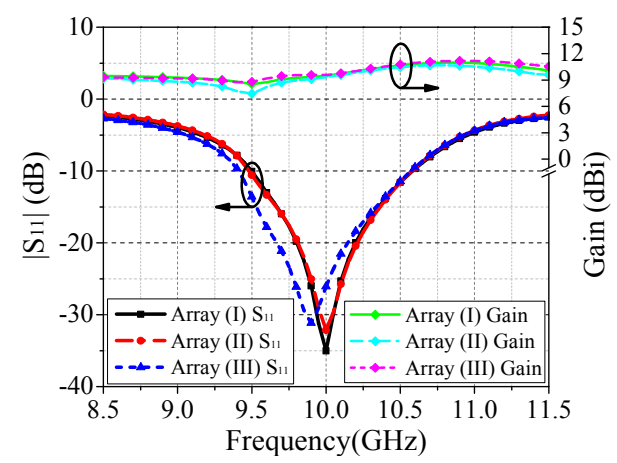

Fig. 8. Simulated reflection coefficient and peak gain.

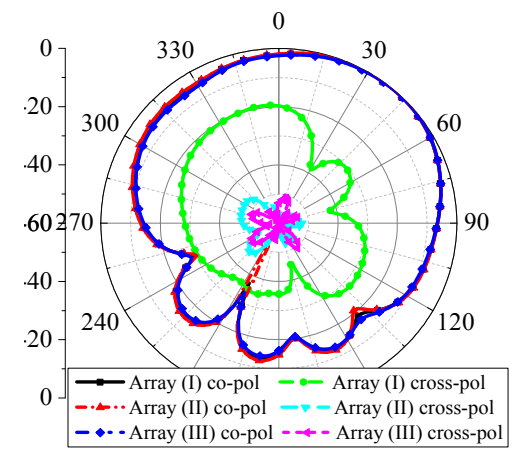

(a)

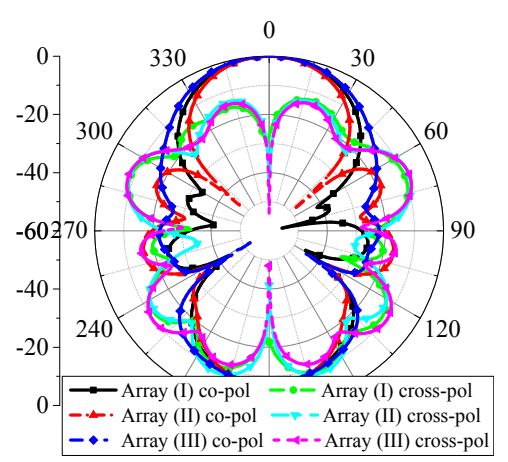

(b)

Fig. 9. Simulated radiation patterns at $10 \mathrm{GHz}$. (a) Elevation plane. (b) Azimuth plane.

bor element as shown in Fig. 10. In other words, the farfield cross-polar component would be eliminated since current components responsible for cross polarization, i.e. $J_{\mathrm{y}}$ components, are all directed in opposite directions [23]. 


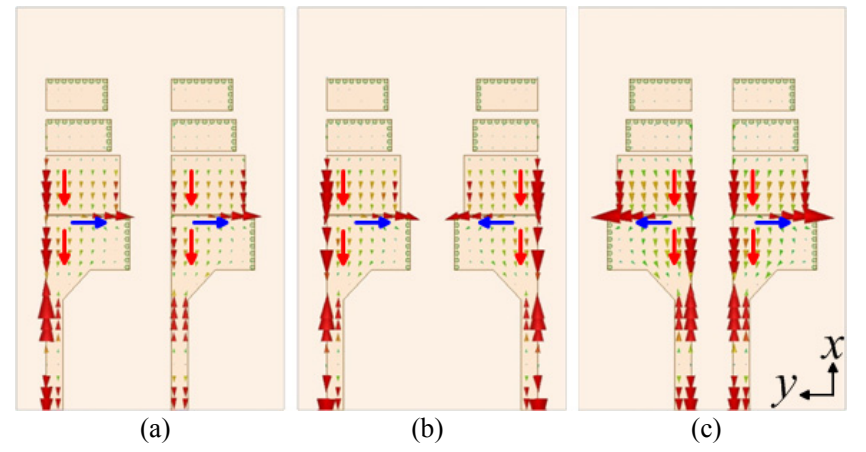

Fig. 10. Comparison of current distributions at $10 \mathrm{GHz}$. (a) Array (I). (b) Array (II). (c) Array (III).

On the contrary, current components responsible for cross polarization have the same direction in array (I), so the far field cross-polar component could not be eliminated and cross polarization still remains high.

\section{Analysis of Four-element Yagi Arrays}

To further investigate the radiation performance of arrays with different arrangements, four-element Yagi arrays are analyzed. Due to the asymmetrical structure, four antenna elements could make up sixteen kinds of arrays with different arrangements. Among the sixteen arrays, six couples are mutually symmetrical. So the number of arrays is reduced to ten. To simplify the analyses, we choose two representative arrays as the research objects. Their configurations are shown in Fig. 11. The array (IV) in Fig. 11(a) is an asymmetrical structure. It is composed by combining array (II) and array (III) together, and the two elements in the middle have the same direction of open sides. In Fig. 11(b), the array (V) is a symmetrical structure and its formation is shown in Fig. 11(c). It is composed by truncating four continuous elements after duplicating array (II) or array (III) along $y$-direction, and adjacent elements have the opposite direction of open sides.

The simulated results of reflection coefficient, peak gain and radiation patterns are presented in Figs. 12 to 15. From these plots, it can be observed that the arrays exhibit a remarkable similarity in bandwidth, peak gain, co-polarization and cross-polarization radiation pattern in azimuth plane, and co-polarization radiation pattern in elevation plane, except for the cross polarization in elevation plane. In the working band, the cross polarization isolation of array $(\mathrm{V})$ is more than $47 \mathrm{~dB}$ within the direction of $0^{\circ} \sim 90^{\circ}$, which is much greater than that of array (IV). Just like the two-element arrays, the reason of low cross polarization is that each element in array (V) has the mirrored current distribution of its neighbor element.

Furthermore, a reasonable corollary could be obtained that arrays with more elements composed by the proposed HMSIW Yagi antennas should have mirrored symmetry that each element is mirrored image of the neighboring one, so as to obtain a lower cross polarization, since the radiated

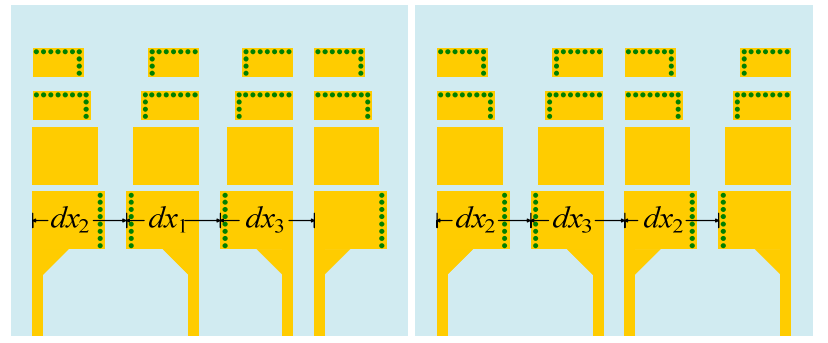

(a)

(b)

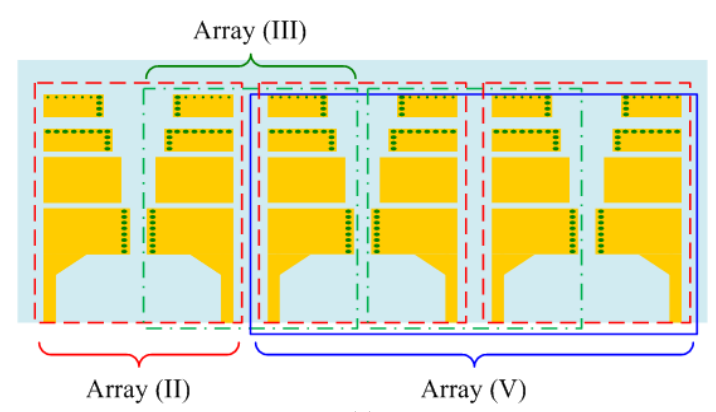

(c)

Fig. 11. Four-element arrays. (a) Array (IV). (b) Array (V). (c) Formation of array (V).

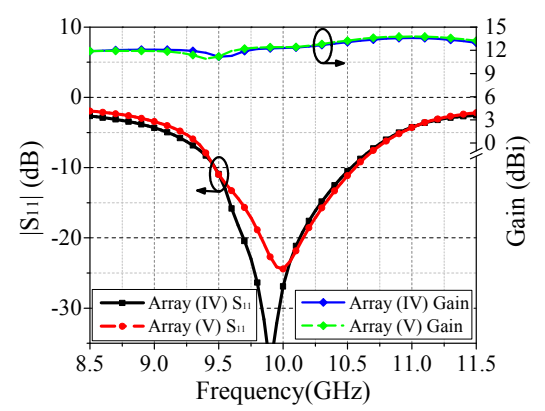

Fig. 12. Simulated reflection coefficient and peak gain.

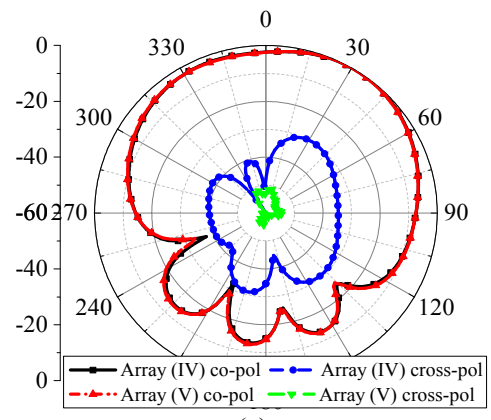

(a)

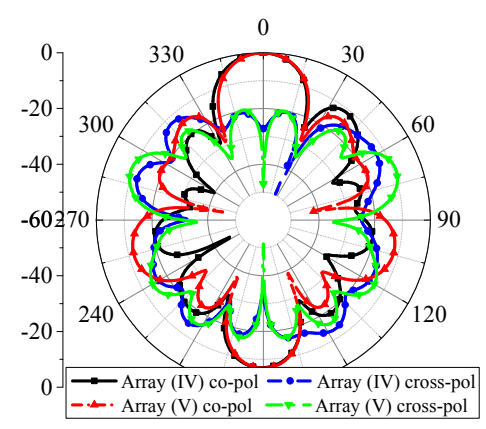

(b)

Fig. 13. Simulated radiation patterns at $9.5 \mathrm{GHz}$. (a) Elevation plane. (b) Azimuth plane. 
fields of every two adjacent elements viewed as a pair will eliminate the far field cross-polar component.

In addition, we should clarify some issues about the radiation performance. Like other Yagi antennas and arrays [1-10], all the presented Yagi antenna and arrays in our work have the tilted beam. Usually, an effective method to adjust the beam steering angle is to change the number of

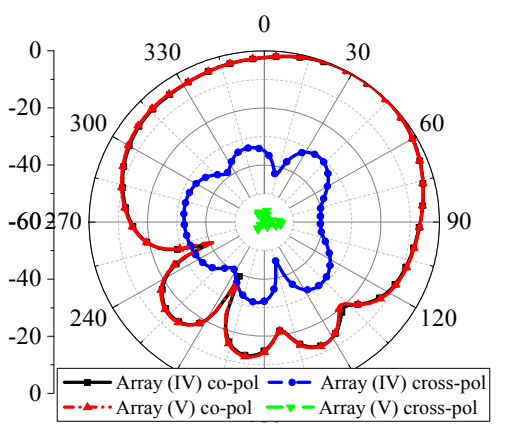

(a)

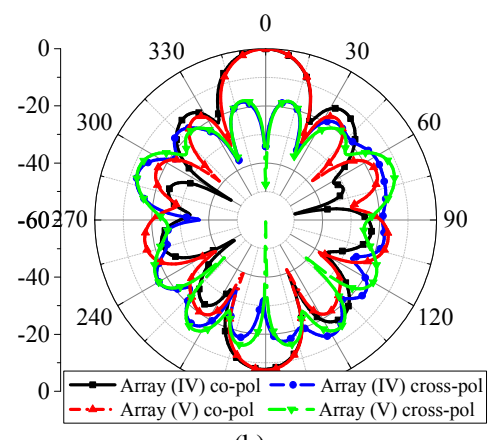

(b)

Fig. 14. Simulated radiation patterns at $10 \mathrm{GHz}$. (a) Elevation plane. (b) Azimuth plane.

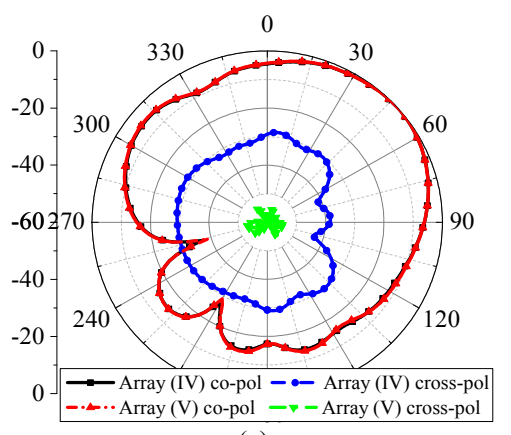

(a)

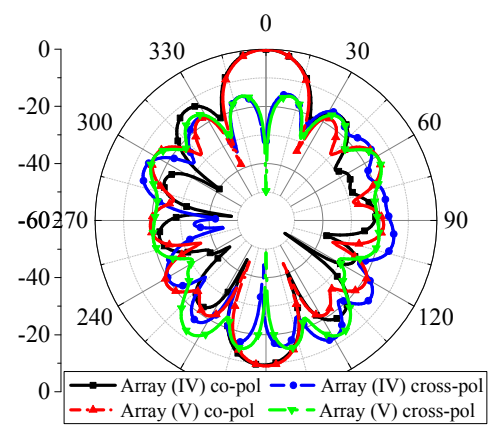

(b)

Fig. 15. Simulated radiation patterns at $10.5 \mathrm{GHz}$. (a) Elevation plane. (b) Azimuth plane. director element according to the principle of field superposition. Another applicative method is to adjust the size of antenna ground plane. Researchers have proved that the Yagi antenna produces a beam radiating certain angle for finite ground plane, while it produces a beam radiating at exactly end-fire for infinite ground plane [4]. The above methods will not change the mirrored arrangement, such as the array (II), array (III) and array (V). So the cross-polarization will still remain at a fairly low level.

To validate the simulation, the four-element array (V) was fabricated and measured in a microwave anechoic chamber as shown in Fig. 16. A vector network analyzer (Agilent N5230C) and a linearly polarized standard-gain horn antenna $(2 \sim 18 \mathrm{GHz})$ were used to constitute a test system. A microstrip $1 \times 4$ Wilkinson power divider was used to feed the array. The power divider works in X-band of $8 \sim 12 \mathrm{GHz}$. The loss and attenuation performance of the power divider is measured to calibrate the measured reflection coefficient of the array. The comparison between simulated and measured results of reflection coefficient and peak gain is shown in Fig. 17. The simulated and measured radiation patterns at $10 \mathrm{GHz}$ are shown in Fig. 18. From these plots, a good agreement between simulation and measurement can be observed. The minor deviation between simulation and measurement is caused by fabrication and measurement.

Finally, performance comparison between different microstrip Yagi antennas and arrays is listed in Tab. 2. Obviously, the intrinsic advantages of the proposed Yagi arrays with mirrored arrangement include not only that they exhibit low profile, high gain and wide bandwidth, but also that the cross-polarization is much lower than others.

\section{Conclusion}

In this paper, low cross polarization Yagi array composed by compact HMSIW Yagi antenna is proposed. Firstly, the HMSIW and magnetic wall are introduced to design the compact Yagi antenna. Results show that the size of driven and director elements are all reduced and the total area is reduced to $1.82 \lambda \times 0.27 \lambda$. Meanwhile the peak gain is about $6.0 \sim 7.9 \mathrm{dBi}$ within the $10.5 \%$ relative bandwidth with VSWR less than 2. Furthermore, two-element and four-element arrays with different arrangements are

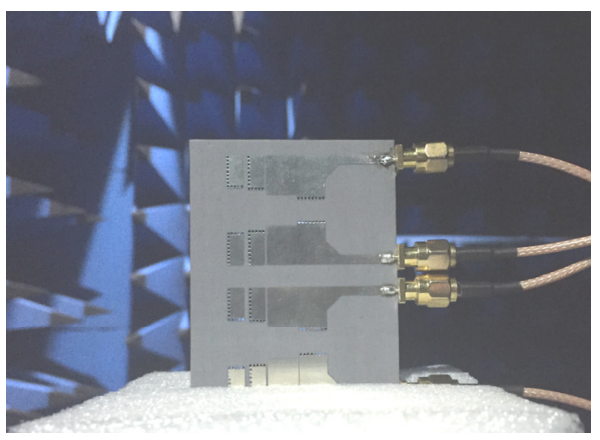

Fig. 16. Measurement in microwave anechoic chamber. 


\begin{tabular}{|c|c|c|c|c|c|}
\hline Antenna & $\begin{array}{c}\text { Cross-polarization } \\
{[\mathbf{d B}]}\end{array}$ & $\begin{array}{c}\text { Gain } \\
{[\mathbf{d B}]}\end{array}$ & $\begin{array}{c}\text { Fractional } \\
\text { Bandwidth }\end{array}$ & $\begin{array}{c}\text { Area } \\
{\left[\lambda^{2}\right]}\end{array}$ & $\begin{array}{c}\text { Profile } \\
{[\lambda]}\end{array}$ \\
\hline Proposed Yagi antenna & -15 & $6.0 \sim 7.9$ & $10.5 \%$ & 1.04 & 0.033 \\
\hline Array (II) & -50 & $8.2 \sim 10.5$ & $10 \%$ & 2.20 & 0.033 \\
\hline Array (V) & -54 & $11.2 \sim 13.4$ & $10 \%$ & 4.26 & 0.033 \\
\hline Ref. [3] & -20 & $9 \sim 11.5$ & $10 \%$ & 3.80 & 0.027 \\
\hline Ref. [4] & -25 & about 10.0 & $13.1 \%$ & 3.82 & 0.027 \\
\hline Ref. [7] & -20 & $15.3 \sim 16.0$ & $5.0 \%$ & 8.20 & 0.027 \\
\hline Ref. [10] & -10 & $6.4 \sim 8.8$ & $3.0 \%$ & 1.11 & 0.013 \\
\hline
\end{tabular}

Tab. 2. Comparison between Yagi antennas and arrays.

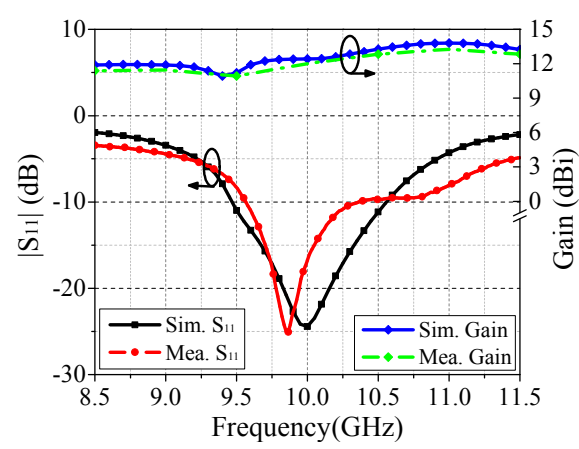

Fig. 17. Simulated and measured reflection coefficient and peak gain.

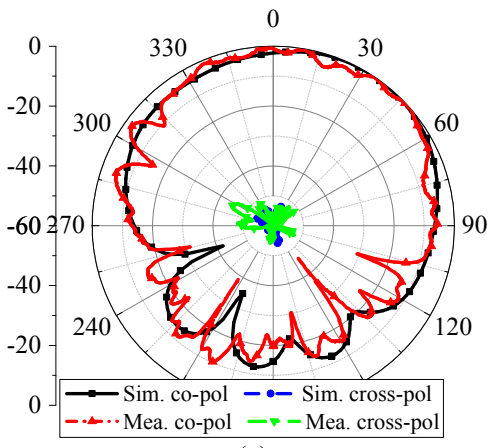

(a)

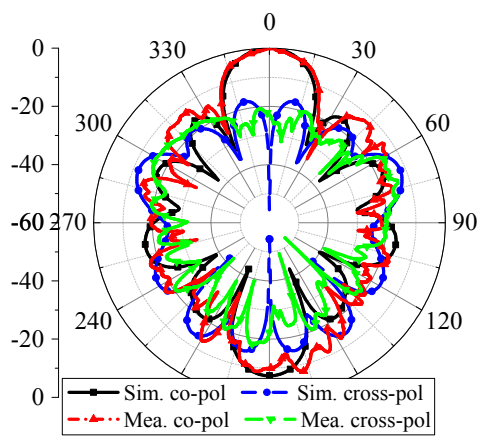

(b)

Fig. 18. Measured radiation patterns.

(a) Elevation plane. (b) Azimuth plane.

analyzed respectively. It has been found that symmetrical arrays with mirrored arrangement, in which each element antenna is the mirrored image of the neighboring one, exhibit a much lower cross polarization because the far field cross-polar component is eliminated. The results provide reference for the design of compact Yagi antenna and the Yagi array with low cross polarization composed by HMSIW elements.

\section{Acknowledgments}

This work is supported by the National Natural Science Foundation of China under Grant No. 61271100, No. 61471389, No. 61501494 and No. 61671464. Authors also thank reviewers for their valuable comments.

\section{References}

[1] HUANG, J. Planar microstrip Yagi array antenna. In International Symposium Digest-Antennas and Propagation, San Jose (USA), 1989, p. 894-897. DOI: 10.1109/APS.1989.134838

[2] PADHI, S. K., BIALKOWSKI, M. E. An X-band aperture-coupled microstrip Yagi array antenna for wireless communications. Microwave and Optical Technology Letters, 1998, vol. 168, no. 5, p. 331-335. DOI: $10.1002 /$ mop. 21344

[3] DEJEAN, G. R., TENTZERIS, M. M. A new high-gain microstrip Yagi array antenna with a high Front-to-Back (F/B) ratio for WLAN and millimetre-wave applications. IEEE Transactions on Antennas and Propagation, 2007, vol. 55, no. 2, p. 298-304. DOI: 10.1109/TAP.2006.889818

[4] LIU, J., XUE, Q. Microstrip magnetic dipole Yagi array antenna with endfire radiation and vertical polarization. IEEE Transactions on Antennas and Propagation, 2013, vol. 61, no. 3, p. 1140-1147. DOI: 10.1109/TAP.2012.2230239

[5] HUANG, J., DENSMORE, A. C. Microstrip Yagi array antenna for mobile satellite vehicle application. IEEE Transactions on Antennas and Propagation, 1991, vol. 39, no. 7, p. 1024-1030. DOI: $10.1109 / 8.86924$

[6] VENKATESAN, J. X-band microstrip Yagi array with dual-offset aperture coupled feed. Microwave and Optical Technology Letters, vol. 48, no. 2, p. 341-344. DOI: 10.1002/mop.21344

[7] DEJEAN, G. R., THAI, T. T., NIKOLAOU, S., et al. Design and analysis of microstrip Bi-Yagi and Quad-Yagi antenna arrays for WLAN applications. IEEE Antennas and Wireless Propagation Letters, 2007, vol. 6, p. 244-248. DOI: 10.1109/LAWP.2007.893104

[8] GRAY, D., LU, J. W., THIEL, D. V. Electronically steerable YagiUda microstrip patch antenna array. IEEE Transactions on Antennas and Propagation, 1998, vol. 46, no. 5, p. 605-608. DOI: $10.1109 / 8.668900$

[9] TSUNEKAWA, K., SAWAYA, K. Compact six-sector antenna employing three intersecting dual-beam microstrip Yagi-Uda arrays with common director. IEEE Transactions on Antennas and Propagation, 2006, vol. 54, no. 11, p. 3055-3062. DOI: 10.1109/TAP.2006.883980

[10] YANG, X.-S., WANG, B.-Z., WU, W., et al. Yagi patch antenna with dual-band and pattern reconfigurable characteristics. IEEE 
Antennas and Wireless Propagation Letters, 2007, vol. 6, p. 168 to 171. DOI: 10.1109/LAWP.2007.895292

[11] YANG, X.-S., WANG, B.-Z., YEUNG, S. H., et al. Circularly polarized reconfigurable crossed-Yagi patch antenna. IEEE Antennas and Propagation Magazine, 2011, vol. 53, no. 5, p. 65 to 80. DOI: 10.1109/MAP.2011.6138429

[12] DESLANDES, D., WU, K. Integrated microstrip and rectangular waveguide in planar form. IEEE Microwave Wireless Component Letters, 2001, vol. 11, no. 2, p. 68-70. DOI: 10.1109/7260.914305

[13] XU, J., HONG, W., TANG, H., et al. Half-mode substrate integrated waveguide (HMSIW) leaky-wave antenna for millimetrewave applications. IEEE Antennas and Wireless Propagation Letters, 2008, vol. 7, p. 85-88. DOI: 10.1109/LAWP.2008.919353

[14] JIN, C., LI, R., ALPHONES, A., et al. Quarter-mode substrate integrated waveguide and its application to antennas design. IEEE Transactions on Antennas and Propagation, 2013, vol. 61, no. 6, p. 2921-2928. DOI: 10.1109/TAP.2013.2250238

[15] SAM, S., LIM, S. Electrically small eighth-mode substrate integrated waveguide antenna with different resonant frequencies depending on rotation of complementary split ring resonator. IEEE Transactions on Antennas and Propagation, 2013, vol. 61, no. 10, p. 4933-4939. DOI: 10.1109/TAP.2013.2272676

[16] SAM, S., LIM, S. Miniaturized circular polarized TE-mode substrate integrated waveguide antenna. IEEE Antennas and Wireless Propagation Letters, 2014, vol. 13, p. 658-661. DOI: 10.1109/LAWP.2014.2313747

[17] JIN, C., LI, R., ALPHONES, A. Compact circularly polarized antenna based on quarter-mode substrate integrated waveguide sub-array. IEEE Transactions on Antennas and Propagation, 2014 vol. 62, no. 2, p. 963-967. DOI: 10.1109/TAP.2013.2291574

[18] XU, F., WU, K. Guided-wave and leakage characteristics of substrate integrated waveguide. IEEE Transactions on Microwave Theory and Techniques, 2005, vol. 53, no. 1, p. 66-73. DOI: 10.1109/TMTT.2004.839303

[19] GARG, R., BAHL, I., BOZZI, M. Microstrip Lines and Slotlines. $3^{\text {rd }}$ ed. Artech House, 2013. ISBN: 9781608075355.

[20] POZAR, D. M. Microwave Engineering. $4^{\text {th }}$ ed. Wiley Publishing, 2004. ISBN: 978-0-470-63155-3

[21] ESQUIUS-MOROTE, M., FUCHS, B., ZÜRCHER, J.-F., et al. A printed transition for matching improvement of SIW horn antennas. IEEE Transactions on Antennas and Propagation, 2013, vol. 61, no. 4, p. 1923-1930. DOI: 10.1109/TAP.2012.2231923

[22] DESLANDES, D., WU, K. Accurate modeling, wave mechanisms, and design considerations of a substrate integrated waveguide. IEEE Transactions on Microwave Theory and Techniques, 2006, vol. 54, no. 6, p. 2516-2526. DOI: 10.1109/TMTT.2006.875807

[23] HASANI, H., KAMYAB, M., MIRKAMALI, A. Low crosspolarization reflectarray antenna. IEEE Transactions on Antennas and Propagation, 2011, vol. 59, p. 1752-1756. DOI: 10.1109/TAP.2011.2123071

\section{About the Authors ...}

Zhao ZHANG was born in Baoji, Shaanxi province, P.R. China in 1990. He received B.S. and M.S. from the Air Force Engineering University, Xi'an China, in 2012 and 2014. He is currently working toward Ph.D. degree at the Information and Navigation College, Air Force Engineering University. His main interests include titled beam antennas, circularly polarized antennas, metamaterial design and RCS reduction.

Xiangyu CAO received the B.Sc and M.A.Sc degrees from the Air Force Missile Institute in 1986 and 1989, respectively. She joined the Air Force Missile Institute in 1989 as an assistant teacher. She became an associate professor in 1996. She received Ph.D. degree in the Missile Institute of Air Force Engineering University in 1999. From 1999 to 2002, she was engaged in postdoctoral research in Xidian University, China. She was a Senior Research Associate in the Department of Electronic Engineering, City University of Hong Kong from Jun. 2002 to Dec. 2003. She is currently a professor of the Air Force Engineering University of CPLA. Her research interests include computational electromagnetic, smart antennas, electromagnetic metamaterial and their antenna applications, and electromagnetic compatibility. 